\title{
Cellulite and Focused Extracorporeal Shockwave Therapy for Non-Invasive Body Contouring: a Randomized Trial
}

\author{
Karsten Knobloch • Beatrice Joest • Robert Krämer • Peter M. Vogt
}

To view enhanced content go to www.dermtherapy-open.com Received: August 20, 2013 / Published online: December 3, 2013

(C) The Author(s) 2013. This article is published with open access at Springerlink.com

\section{ABSTRACT}

Introduction: Focused extracorporeal shockwave therapy (ESWT) has been demonstrated to improve wound healing and skin regeneration such as in burn wounds and scars. We hypothesized that the combination of focused ESWT and a daily gluteal muscle strength program is superior to SHAM-ESWT and gluteal muscle strength training in moderate to severe cellulite.

ClinicalTrials.gov identifier: NCT00947414.

Electronic supplementary material The online version of this article (doi:10.1007/s13555-013-0039-5) contains supplementary material, which is available to authorized users.

K. Knobloch $(\square)$

SportPraxis, Heiligerstr. 3, Hannover, Germany e-mail: professor.knobloch@sportpraxis-knobloch.de

B. Joest · R. Krämer · P. M. Vogt

Department of Plastic, Hand and Reconstructive Surgery, Hannover Medical School, Hannover, Germany

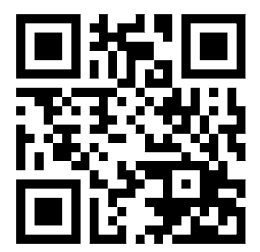

Enhanced content for this article is available on the journal web site: www.dermtherapy-open.com
Methods: This was a single-center, doubleblinded, randomized-controlled trial. For allocation of participants, a 1:1 ratio randomization was performed using opaque envelopes for the concealment of allocation. Eligible patients were females aged 18-65 years with cellulite. The primary outcome parameter was the photo-numeric Cellulite Severity Scale (CSS) determined by two blinded, independent assessors. The intervention group (group A) received six sessions of focused ESWT $(2,000$ impulses, $0.35 \mathrm{~mJ} / \mathrm{mm}^{2}$, every $1-2$ weeks) at both gluteal and thigh regions plus specific gluteal strength exercise training. The control group (group B) received six sessions of SHAMESWT plus specific gluteal strength exercise training.

Results: The CSS in group A was 10.9 \pm 3.8 (mean \pm SE) before intervention and $8.3 \pm 4.1$ after 12 weeks $(P=0.001,2.53$ improvement, $95 \%$ confidence interval (CI) 1.43-3.62). The CSS in group B was $10.0 \pm 3.8$ before intervention and $10.1 \pm 3.8$ after 12 weeks $(P=0.876,95 \%$ CI 1.1-0.97). The change of the CSS in group A versus group B was significantly different $(P=0.001,-24.3$ effect size, $95 \% \mathrm{CI}-36.5$ to -12.1$)$. 
Conclusion: The combination of non-invasive, focused ESWT $\left(0.35 \mathrm{~mJ} / \mathrm{mm}^{2}, 2,000\right.$ impulses, 6 sessions) in combination with gluteal strength training was superior to gluteal strength training and SHAM-ESWT in moderate to severe cellulite in terms of the CSS in a 3-month perspective. Long-term results have to be evaluated in terms of the sustainability of these effects.

Keywords: Aesthetics; Body contouring; Cellulite; Dermatology; Extracorporeal shock wave therapy; Strength training

\section{INTRODUCTION}

Cellulite is a widespread problem involving the buttocks and thighs of the female-specific anatomy [1]. The higher number of fat cells stored in female fatty tissue in contrast to males, the gender-specific dimorphism with subdermal septae orientated orthogonally toward the skin, and the aging process of connective tissue lead to an imbalance between lipogenesis and lipolysis with subsequent large fat cells bulging the skin [1]. Recently, a case-control study in 15 lean women suffering from cellulite, and ageand body mass index (BMI)-matched controls identified significantly reduced adiponectin expression using reverse transcription polymerase chain reaction among the celluliteaffected patients [2]. Cellulite appears to potentially impair quality of life of affected females substantially. It appears that younger females affected by cellulite suffer more in terms of impaired quality of life than more mature females [3].

Non-randomized clinical data suggest that extracorporeal shock wave therapy (ESWT) is beneficial in terms of improved skin elasticity and revitalizing dermis in females with cellulite $[4,5]$. Potentially, a direct effect on the associated lymphedema is a further potential consequence of ESWT application in cellulite. A recent Korean prospective clinical trial evaluated the effect of four ESWT sessions $\left(0.056-0.068 \mathrm{~mJ} / \mathrm{mm}^{2}, 2,000\right.$ impulses, ESWT device from Dornier AB2) within 2 weeks in patients suffering from secondary lymphedema [6]. Both the circumference and the thickness of the skin fold of the affected region were significantly reduced by as much as $37 \%$ in line with a pain reduction on the visual analogue scale [6]. In systemic sclerosis, ESWT is able to again improve pain and the Rodnan skin score for skin wellness [7].

To date, a limited number of non-controlled studies (two Level III $[4,8]$ and two Level IV studies $[9,10])$ examined the effect of ESWT on cellulite with various outcome measures (Table 1).

Recently, a small size $(n=25)$ randomizedcontrolled trial (RCT) with large confidence intervals (CIs) has been published (level 2 evidence) [11]. The trial involved six sessions over 4 weeks using the STORZ D-ACTOR ${ }^{\circledR} 200$ by Storz Medical (Tägerwilen, Switzerland) improved depressions, elevations, roughness, and elasticity within 3 months.

Beyond the aforementioned RCT [11], with small sample size and large confidence intervals, we do not have any high-level $1 \mathrm{~b}$ evidence to support the use of focused ESWT for non-invasive body contouring in cellulite. In addition, we do not know whether or not and if, to what extent the validated photo-numeric Cellulite Severity Scale (CSS) is changed by six sessions of focused ESWT. Currently, we do not have any high-level $1 \mathrm{~b}$ evidence regarding the effect of gluteal home-based strength training with or without focused ESWT on the clinical outcome in cellulite in terms of digital images, microcirculation and patient self-reported assessment. Given these facts we sought to 


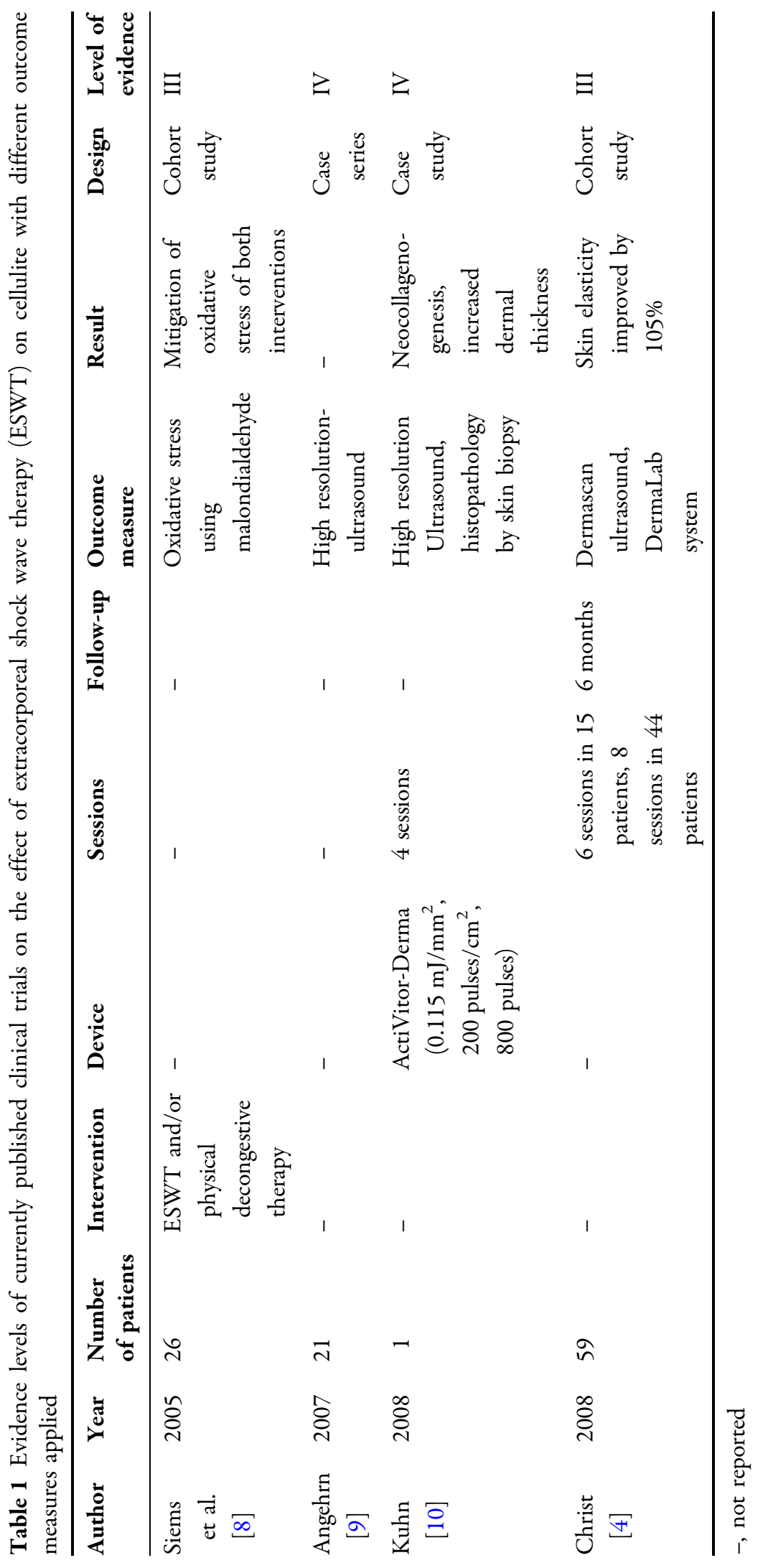


overcome these issues and performed a doubleblinded, RCT providing level $1 \mathrm{~b}$ evidence on the use of focused ESWT in addition to daily gluteal strength training in various degrees of cellulite. We hypothesized that the combination of ESWT and a daily gluteal muscle strength program is superior to SHAM-ESWT and a gluteal muscle strength program in cellulite.

\section{METHODS}

The study protocol was composed according to the most recent CONSORT 2010 recommendations for transparent reporting of RCTs $[12,13]$. The study protocol according to the CONSORT recommendations has been published previously [14].

\section{Ethics and Trial Registration}

This RCT was approved in May 22, 2009 by the ethics institutional review board at Hannover Medical School, Germany, under the German title "Stosswellentherapie und Krafttraining zur Therapie der Cellulite-eine randomsiertkontrollierte Studie" (Nr. 5206). The study is internationally registered at ClinicalTrials.gov with ClinicalTrials.gov identifier: NCT00947414.

All procedures followed were in accordance with the ethical standards of the responsible committee on human experimentation (institutional and national) and with the Helsinki Declaration of 1975, as revised in 2000 and 2008. Informed consent was obtained from all patients for being included in the study and for the publication of patient photographs.

\section{Study Design}

This was a single-center, double-blinded, RCT with a 1:1 parallel group randomization.

\section{Participants}

The mean age of the enrolled participants was 41.4 years in the intervention group and 45.0 years in the control group. BMI (mean $\pm \mathrm{SE}$ ) was $24.2 \pm 3.2 \mathrm{~kg} / \mathrm{m}^{2}$ in the intervention group and $25.3 \pm 4.5 \mathrm{~kg} / \mathrm{m}^{2}$ in the control group.

Eligible patients were females aged between 18 and 65 years with documented cellulite $0^{\circ}$ to $3^{\circ}$ according to the Nürnberger Müller score [1]. Exclusion criteria were the following: suspected or evident pregnancy, no cellulite, no informed consent, and age under 18 years or above 65 years. Patients were recruited by advertisements in local regional newspapers and via the Internet. The patient enrollment flow chart according to the CONSORT statement is outlined in Fig. 1.

\section{Interventions}

In CelluShock-2009 patients were randomly assigned with a 1:1 ratio to either ESWT with $0.35 \mathrm{~mJ} / \mathrm{mm}^{2}$ in the intervention group or $0.01 \mathrm{~mJ} / \mathrm{mm}^{2}$ in the SHAM-ESWT group. Both groups additionally participated in a homebased, daily gluteal thigh exercise program.

The intervention group received six sessions of ESWT (every 1-2 weeks) with focused shock waves (2,000 impulses, $0.35 \mathrm{~mJ} / \mathrm{mm}^{2}$, Fig. 2) plus home-based, daily gluteal strength exercises (Figs. 3, 4). The control group received six sessions of SHAM-ESWT $(2,000$ impulses, $0.01 \mathrm{~mJ} / \mathrm{mm}^{2}$, every $1-2$ weeks) plus home-based, daily gluteal strength exercises. Extracorporeal shock wave therapy was applied using a STORZ focused Duolith machine (Taegerwilen, Suisse) as acoustic wave treatment.

In order to increase the motivation of the participating females, especially in terms of 


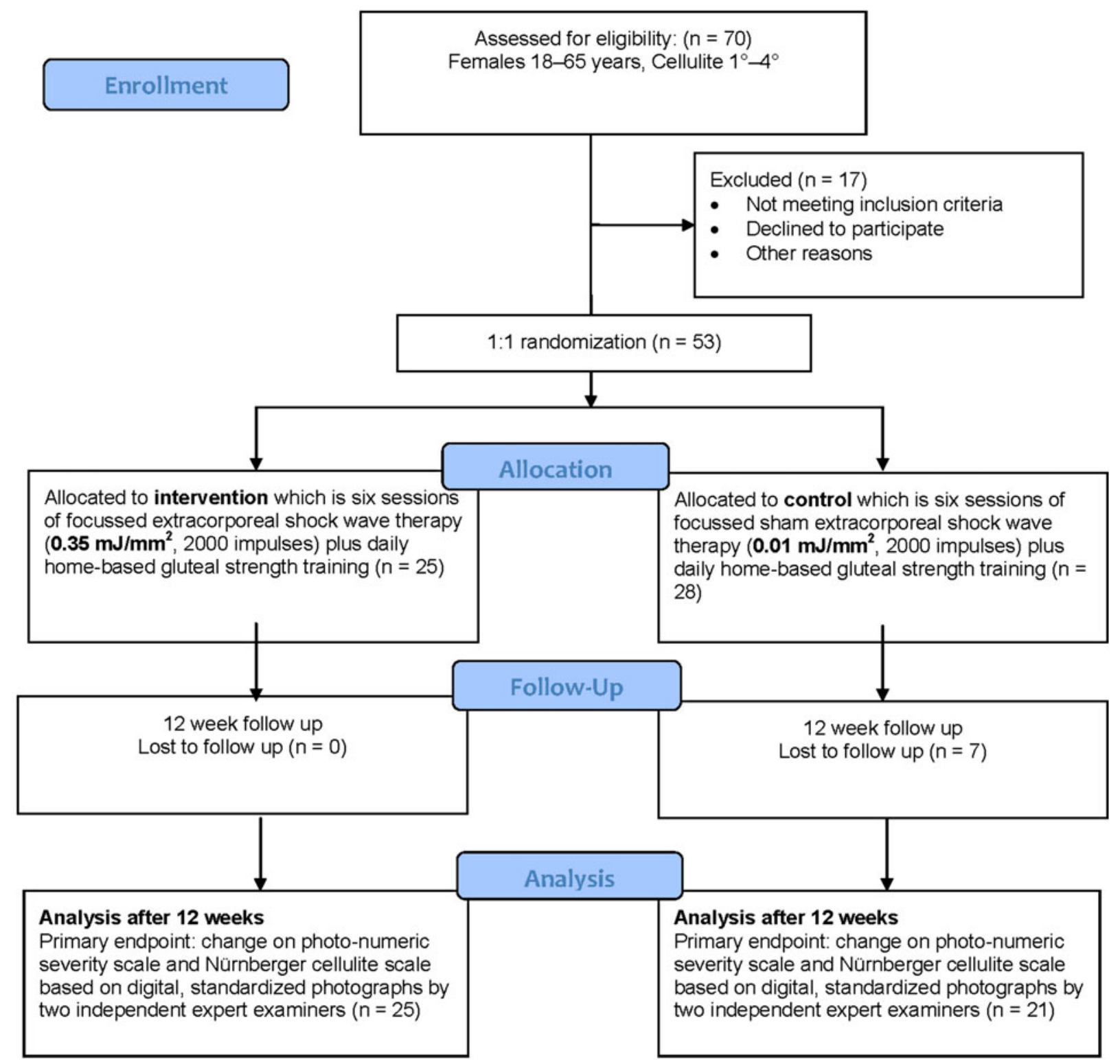

Fig. 1 CONSORT patient flow chart. Modified with permission from Knobloch et al. [14]

follow-up, we added a daily, home-based gluteal strength exercise program. Twice a day (in the morning and the evening), two different exercises focusing on the gluteal muscles involving the piriformis, the gemelli, and the gluteal muscles were performed with 15 repetitions for each leg (Figs. 1, 2). The compliance to the daily gluteal workout program was noted in a exercise log to improve and supervise participants' compliance, respectively.

\section{Primary and Secondary Outcome Measures}

The primary endpoint, with respect to efficacy of the combined ESWT and gluteal strength exercises versus SHAM-ESWT and the same 


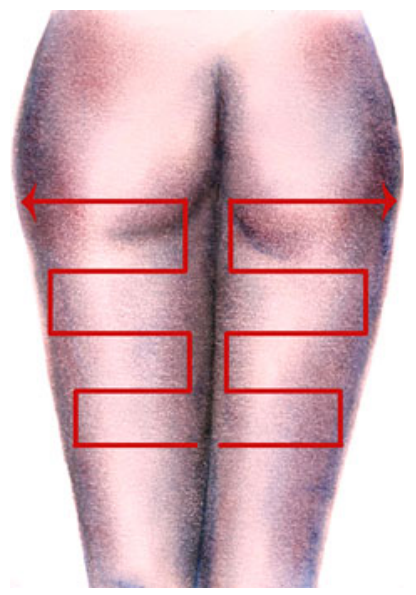

Fig. 2 Placement of the shockwave probe from distal to proximal on both thighs
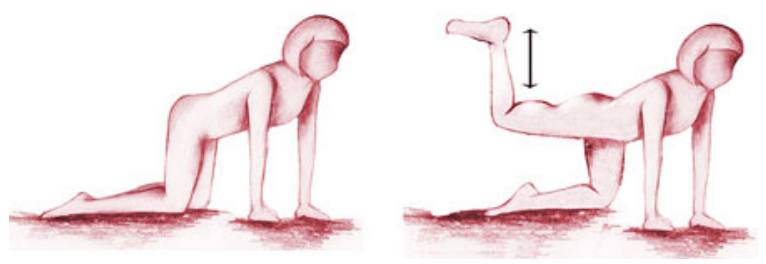

Fig. 3 First exercise (15 repetitions per leg twice a day over 12 weeks)
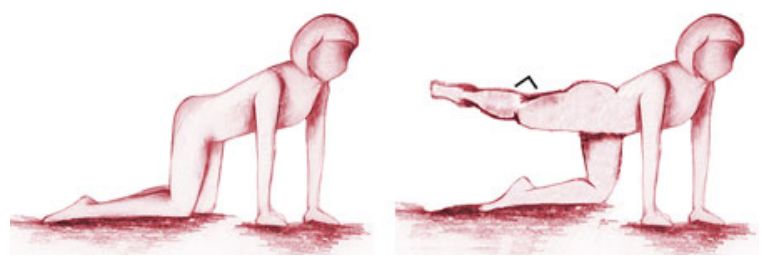

Fig. 4 Second exercise (15 repetitions per leg twice a day over 12 weeks)

gluteal strength exercise program, was the change on digital photographs 3 months after the last ESWT treatment assessed by the validated CSS [15]. This provided reliable, comprehensive, and reproducible results. Cellulite severity may be classified according to the result of this assessment in the CSS in three degrees, as described in Table 2.

The classification was performed based on standardized photographs taken by a professional medical photographer at baseline
Table 2 Cellulite Severity Scale of mild, moderate or severe degree

\begin{tabular}{ll}
\hline Cellulite Severity Scale & New classification \\
\hline $1-5$ & Mild \\
$6-10$ & Moderate \\
$11-15$ & Severe \\
\hline
\end{tabular}

and 12 weeks after the last ESWT treatment in both groups. The assessment of the anonymous digital images was carried out by two blinded assessors who were not aware of either the study arm or the fact that it is a baseline or a follow-up photograph taken 12 weeks after the last ESWT treatment in both groups.

In order to overcome the problems of interpretation associated with multiplicity of analyses we decided to choose the aforementioned clinical endpoint which is a visual one as the primary endpoint and results as secondary endpoints in CelluShock.

Secondary endpoints of the CelluShock RCT were as follows: change of circumference of the thigh $(\mathrm{cm})$, skin elasticity using the Cutometer ${ }^{\circledR}$ (Cutometer MPA 580, Kosmetik Konzept KOKO GmbH \& Co KG, Leichlingen, Germany) [1619], and self-assessment of the success on a visual analogue scale $0-10 \quad(0=$ no change, 10 = fully satisfied).

All patients were measured at baseline and after 12 weeks regarding the primary and all secondary endpoints.

\section{Power Calculation}

To detect at least a change of two points in the CSS of cellulite, with a two-sided, 5\% significance with an $80 \%$ power, a sample size of 26 participants with an estimated drop-out rate of $15 \%$ was calculated. This was done prior to the start of trial. 


\section{Randomization and Allocation Sequence}

For allocation of participants, a 1:1 ratio randomization was performed using opaque envelopes for the concealment of allocation. The allocation sequence was concealed from the researcher (BJ) enrolling and assessing participants in sequentially numbered, opaque, sealed envelopes [20].

\section{Blinding}

Blinding was achieved for all participants enrolled in the trial, the photographer taking the digital images for the primary outcome measure, the two assessors of the outcome measures, all additional health care providers, and for the analyst from the biometrical department. Only one researcher (BJ) was aware of the group assignment performing the randomization and the ESWT.

The assessment of the primary and secondary outcomes was performed by blinded assessors independently from each other, unaware whether the digital image displayed was before or after therapy or with group (intervention or control group) was randomized.

\section{Statistical Analysis}

The primary endpoint was change of CSS assessed on digital, standardized photographs by two independent expert examiners. Student's $t$ test was applied for parametric data, the Wilcoxon test for non-parametric data, and a level of $P<0.05$ was reported as significant. An intention-to-treat analysis was applied. SPSS (IBM Corp., New York, USA) was used to carry out the analysis.

\section{CONSORT Flow Chart}

Figure 1 highlights the patient flow throughout the CelluShock-2009 RCT (Fig. 1).

\section{RESULTS}

\section{Primary Outcome Measure-Cellulite Severity Scale}

The CSS (mean \pm SE) in the intervention group was $10.9 \pm 3.8$ before and $8.3 \pm 4.1$ after the combined ESWT and strength exercise
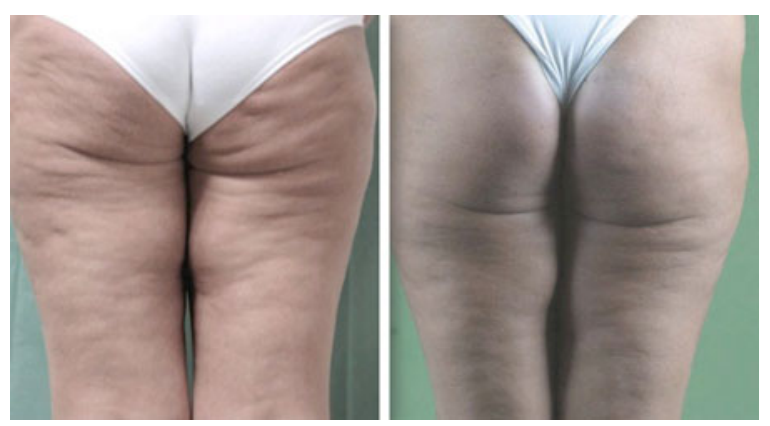

Fig. 5 Improvement of the Cellulite Severity Score from 15 to 7 in a female patient suffering from cellulite before and 3 months after six sessions of focused extracorporeal shockwave therapy $\left(0.35 \mathrm{~mJ} / \mathrm{mm}^{2}\right)$
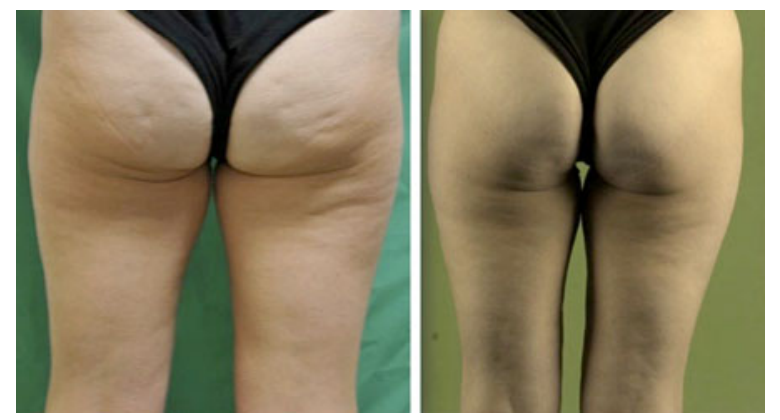

Fig. 6 Improvement of the Cellulite Severity Score from 12 to 2 in a female patient suffering from cellulite before and 3 months after six sessions of focused extracorporeal shockwave therapy $\left(0.35 \mathrm{~mJ} / \mathrm{mm}^{2}\right)$ 

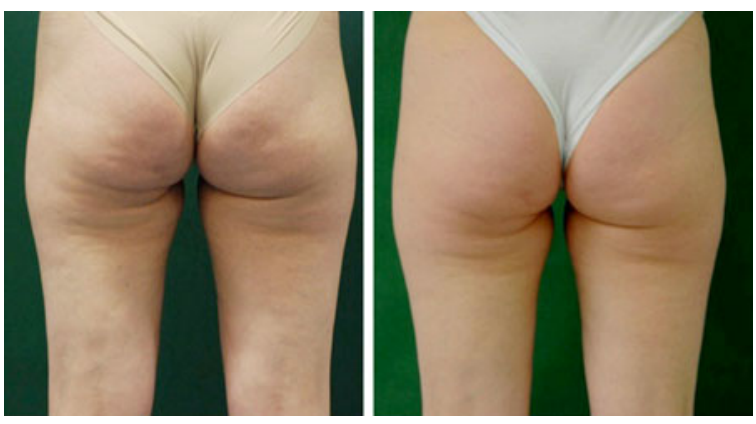

Fig. 7 Improvement of the Cellulite Severity Score from 10 to 6 in a female patient suffering from cellulite before and 3 months after six sessions of focused extracorporeal shockwave therapy $\left(0.35 \mathrm{~mJ} / \mathrm{mm}^{2}\right)$
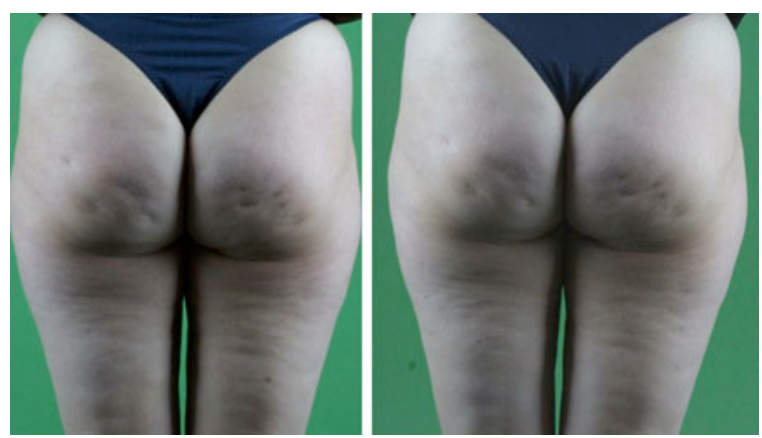

Fig. 8 No significant improvement of the Cellulite Severity Score from 13 to 11 in a female patient suffering from cellulite before and 3 months after six sessions of sham extracorporeal shockwave therapy $\left(0.01 \mathrm{~mJ} / \mathrm{mm}^{2}\right.$, control group)
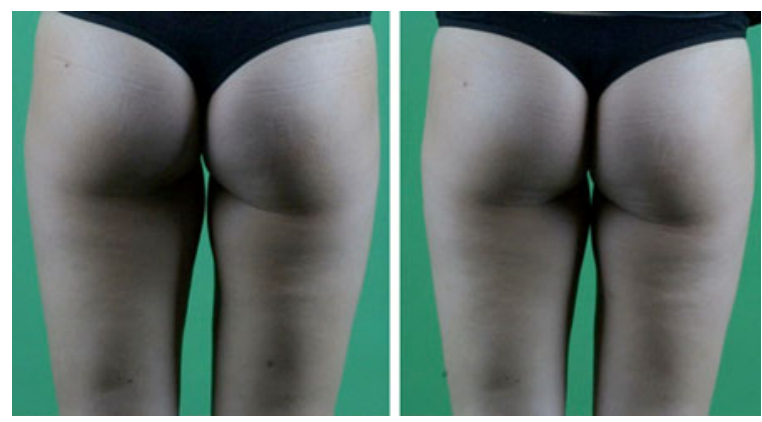

Fig. 9 No improvement of the Cellulite Severity Score from 4 to 5 in a female patient suffering from cellulite before and 3 months after six sessions of sham extracorporeal shockwave therapy $\left(0.01 \mathrm{~mJ} / \mathrm{mm}^{2}\right.$, control group)

intervention $(P=0.001,2.53$ improvement (+24\%), 95\% CI 1.43-3.62) (Figs. 5, 6, 7, 8, 9, 10; Table 3). The CSS in the control group was

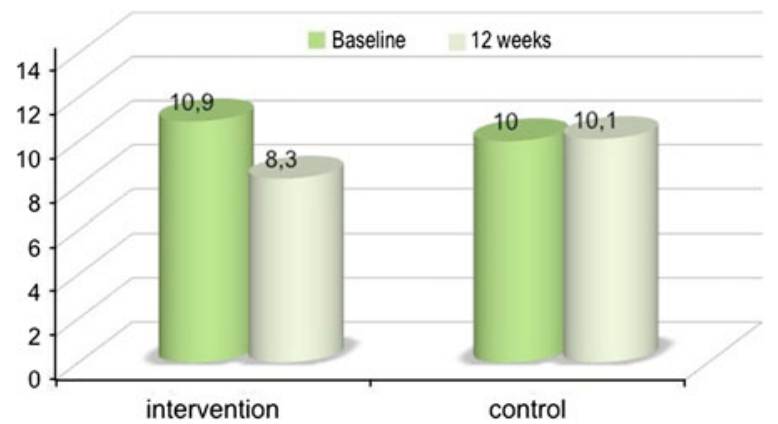

Fig. 10 Change of the Cellulite Severity Score before and 3 months after six sessions of either focused extracorporeal shockwave therapy (ESWT) $\left(0.35 \mathrm{~mJ} / \mathrm{mm}^{2}\right.$, intervention group) or SHAM-ESWT $\left(0.01 \mathrm{~mJ} / \mathrm{mm}^{2}\right.$, control group)

$10.0 \pm 3.8$ before and $10.1 \pm 3.8$ after the SHAM-ESWT and strength exercise intervention ( $P=0.876,95 \% \mathrm{CI}-1.1$ to 0.97$)$. The change of the CSS in the intervention group versus the control group was significantly different $(P=0.001,-24.3$ effect size, $95 \%$ -36.5 to -12.1$)$.

The results for the five items of the CSS, the cutometer data, and the thigh circumferences are reported below; all values are given as mean \pm SE unless otherwise stated.

\section{Number of Depressions}

The number of depressions in the intervention group was $2.2 \pm 0.8$ at baseline and $1.8 \pm 0.9$ at follow-up $(P=0.001$, improvement $0.41,95 \% \mathrm{CI}$ $0.17-0.65)$. The number of depressions in the control group was $2.0 \pm 0.8$ at baseline and $2.0 \pm 0.7$ at follow-up $(P=0.534,95 \% \mathrm{CI}-0.30$ to 0.16 ). The change of the number of depressions in the intervention versus the control group was significantly different $(P=0.012,-20.0$ effect size, $95 \%$ CI -34.8 to -4.4 ).

\section{Depth of Depressions}

The depth of depressions in the intervention group was $2.2 \pm 0.8$ at baseline and $1.6 \pm 0.8$ at 
Table 3 Number of patients in each group according to the Cellulite Severity Scale in the intervention and the control group prior and after the intervention

\begin{tabular}{llllll}
\hline Cellulite Severity Scale & \multicolumn{2}{l}{ Intervention group } & & \multicolumn{2}{l}{ Control group } \\
\cline { 2 - 3 } & Baseline & Post-intervention & & Baseline & Post-intervention \\
\hline $1-5$ (mild) & 4 & 6 & 3 & 2 \\
$6-10$ (moderate) & 8 & 12 & 9 & 12 \\
$11-15$ (severe) & 13 & 7 & 9 & 7 \\
\hline
\end{tabular}

follow-up ( $P=0.001,0.61$ improvement, 95\% CI 0.39-0.84). The depth of depressions in the control group was $2.0 \pm 0.8$ at baseline and $2.0 \pm 0.7$ at follow-up $(P=1.0,95 \% \mathrm{CI}-0.24$ to $0.24)$. The change of the depth of depressions in the intervention group versus the control group was significantly different $(P=0.001,-31.3$ effect size, $95 \%$ CI -46.0 to -16.6$)$.

\section{Morphological Appearance of Skin Surface Alterations}

The morphological appearance of skin surface alterations in the intervention group was $2.2 \pm 0.8$ at baseline and $1.6 \pm 0.8$ at follow-up ( $P=0.001,0.6$ improvement, 95\% 0.36-0.8). The morphological appearance of skin surface alterations in the control group was $1.9 \pm 0.8$ at baseline and $1.9 \pm 0.6$ at follow-up $(P=0.837$, $95 \%$ CI -0.20 to 0.25 ). The change of the morphological appearance of skin surface alterations in the intervention group versus the control group was significantly different $(P=0.007,-16.6$ effect size, $95 \%$ CI -28.7 to $-4.6)$.

\section{Grade of Laxity, Flaccidity or Sagging Skin}

The grade of laxity, flaccidity or sagging skin in the intervention group was $2.2 \pm 0.8$ at baseline and $1.6 \pm 0.8$ at follow-up $(P=0.001,0.5$ improvement, $95 \%$ CI $0.27-0.73$ ). The grade of laxity, flaccidity or sagging skin in the control group was $2.0 \pm 0.9$ at baseline and $2.1 \pm 0.8$ at follow-up ( $P=0.516,95 \% \mathrm{CI}-0.34$ to 0.17$)$. The change of the grade of laxity, flaccidity or sagging skin in the intervention group versus the control group was significantly different $(P=0.001,-25.1$ effect size, $95 \% \mathrm{CI}-39.6$ to $-10.6)$.

\section{Classification Scale by Nürnberger and Müller}

The classification scale by Nürnberger and Müller in the intervention group was $2.2 \pm 0.8$ at baseline and $1.8 \pm 0.9$ at follow-up ( $P=0.001,0.5$ improvement, 95\% CI 0.3-0.8). The classification scale by Nürnberger and Müller in the control group was $2.1 \pm 0.8$ at baseline and $2.1 \pm 0.7$ at follow-up $(P=1.0$, $95 \%$ CI -0.22 to 0.22 ). The change of the classification scale by Nürnberger and Müller in the intervention group versus the control group was significantly different $(P=0.043,-24.4$ effect size, $95 \%$ CI -37.7 to -11.1 ).

\section{Change of Circumference of the Thigh and Body Mass Index}

The change of thigh circumference in the intervention group was $61.5 \pm 6.2 \mathrm{~cm}$ at baseline to $61.0 \pm 5.9 \mathrm{~cm}$ at follow-up $(P=0.760,95 \% \mathrm{CI}-2.91$ to 3.97$)$. There was 
no change of thigh circumference in the control group $(61.6 \pm 6.9 \mathrm{~cm})$ at baseline versus followup $(61.6 \pm 6.9 \mathrm{~cm} ; P=0.996 ; 95 \% \mathrm{CI}-4.28$ to 4.31). Pre- and post-treatment body weight index did not change in either group significantly beyond $3 \%$.

\section{Skin Elasticity Using the Cutometer ${ }^{\circledR}$}

The skin elasticity in the intervention group was $14.1 \pm 2.5 \%$ at baseline and $14.1 \% \pm 1.6$ at follow-up ( $P=0.963 ; 95 \% \mathrm{CI}-1.22$ to 1.16$)$.

The skin elasticity in the control group was $14.4 \% \pm 1.8$ at baseline and $14.1 \% \pm 1.9$ at follow-up ( $P=0.676 ; 95 \% \mathrm{CI}-0.91$ to 1.38$)$.

\section{DISCUSSION}

The combination of focused ESWT $(0.35 \mathrm{~mJ} /$ $\mathrm{mm}^{2}, \quad 2,000$ impulses, 6 sessions) in combination with gluteal strength training was superior to a gluteal strength training alone in terms of the CSS in a 3-month perspective. The significant mean improvement was $24 \%$ in the intervention group in contrast to the control group, a clinically meaningful difference. Second, the SHAM-ESWT and the gluteal strength training were not able to change the CSS.

The strengths of this double-blinded, randomized clinical trial are the independent assessment by two expert examiners who were blinded to both the patients and the group allocation. Both experts assessed the digitalized standardized photographs independently and the mean of both assessments was applied. Second, this is the first registered, double-blinded, randomized clinical trial to assess the effects of a gluteal strength training and the combination with focused ESWT. Third, standardized photographs were taken by a clinical photographer independently from the study team. Fourth, the control group received SHAMESWT $\left(0.01 \mathrm{~mJ} / \mathrm{mm}^{2}, 2,000\right.$ impulses, 6 sessions $)$ which did not appear to have any clinical effect in terms of the CSS at all, with identical values before and after the intervention.

However, to date we cannot estimate the long-term efficacy and sustainability of the aforementioned clinical effects in a perspective of one or more years. It is possible that, after a year, an additional treatment might be warranted, such as a touch-up procedure. In our personal experience, select cases might benefit even longer than 1 year from a set of six focused shockwave sessions, but this is only a non-controlled observation.

The results of this randomized clinical study should be discussed in detail. The CSS is a validated photo-numeric Cellulite Severity Scale, which has been published in 2009 by Dr. Hexsel and coworkers [15]. Beyond the wellknown Nürnberger and Müller score ranging from $0^{\circ}$ to $3^{\circ}$, this validated score appears to better reflect even modest to small changes of a given therapeutic intervention. The CSS has a high intraclass correlation coefficient of 0.9 or more and ranges from 1 to 15 . Three clinical cellulite severity grades have been proposed by Hexsel et al. [15] (Table 2). Given our patients, we included the majority of patients with moderate to severe degrees of cellulite. This is partially reflected by the mean age beyond 40 years and the BMI beyond $24.2 \mathrm{~kg} / \mathrm{m}^{2}$ in both groups.

As far as the underlying mechanisms of the evident improvements in the CSS are concerned, a "mechanical" response might be evident as well as a "regenerative" response of the afflicted skin.

In terms of the "mechanical" perspective, one might speculate that the focused extracorporeal shockwave has somewhat 
disrupted either the fat components or the septae or both, which might lead to a smoothening of the afflicted skin. MR imaging has shown that fibrous septa are visualized in $97 \%$ of the area with cellulite depressions, which are markedly thickened in cellulite afflicted areas [21]. Shockwave energy might have weakened the fibrous septae and thus the afflicted skin became smoother.

Reduction of lymphedema is a second potential underlying mechanism. Recently, a significant reduction of lymphedema was reported clinically following four ESWT sessions in females with secondary lymphedema following breast cancer treatment [6]. In animal experiments ESWT and the vascular endothelial growth factor (VEGF-C) hydrogel appear to exert a synergistic effect in promoting lymphangiogenesis [22].

On the other hand, ESWT might somewhat influence mesenchymal stem cells. There is evolving experimental data suggesting that shockwave therapy activation pathways in adipose-derived stem cells [23]. Clinically, diseased skin appears to normalize following shockwave treatment such as in progressive systemic sclerosis with an up regulation of endothelial progenitor cells and circulating endothelial cells [24].

Energy flux density of the focused ESWT is another issue to concern. We used low to medium energy flux densities of $0.35 \mathrm{~mJ} / \mathrm{mm}^{2}$ with 1,000 impulses on each thigh with $4 \mathrm{~Hz}$. To date, we do not know in controlled trials whether potentially higher energy flux densities such as up to $1.24 \mathrm{~mJ} / \mathrm{mm}^{2}$ might be even more beneficial in terms of the potential disruption of the fibrous septae in the cellulite-afflicted areas. On the other hand, stem-cell activation might be achieved by rather low-energy flux densities in regard of the aforementioned potential underlying "regenerative" mechanisms.
To date, only small size, controlled trials with wide CIs have been published [7, 25]. We tried to overcome the methodological shortcomings of previous trials in CelluShock2009. In regard to different techniques, there are evolving clinical data that, for example, lowlevel laser therapy with $532 \mathrm{~nm}$ wave lengths appears to improve cellulite in a double-blind, placebo-controlled, randomized trial [26]. 1,064 nm Nd:YAG laser appears to improve mild to moderate cellulite also [27]. Radiofrequency is able to reduce cellulite in a randomized trial [28].

\section{Limitations}

Given our randomized, double-blinded clinical trial, some limitations have to be considered when interpreting our data. First, the extent of cellulite reflected by digital unprocessed images assessed by two independent examiners was chosen to overcome some types of biases. However, a digital image does not necessarily reflect or even replace a clinical examination including a pinch test. However, we sought to address as objective as possible the outcome based on digital images. Those images were produced by a clinical plastic surgical professional photographer under the very same circumstances to overcome issues such as angle of the photograph, lighting, among others. To date, we can only report the shortterm results 3 months following ESWT. We do not know the long-term effects in terms of efficacy and sustainability of six sessions of focused ESWT in cellulite to date.

\section{CONCLUSION}

The combination of focused ESWT $(0.35 \mathrm{~mJ} /$ $\mathrm{mm}^{2}, 2,000$ impulses, 6 sessions) with gluteal 
strength training was superior to SHAM-ESWT and gluteal strength training in moderate to severe cellulite in terms of the CSS in a RCT. The mean improvement was $24 \%$ in the intervention in contrast to the control group. Second, the SHAM-ESWT and gluteal strength training were not able to change the CSS in a 3-month perspective. Long-term data are warranted to elucidate the sustainability of the aforementioned clinical effects.

\section{ACKNOWLEDGMENTS}

Prof. Knobloch is the guarantor for this article and takes responsibility for the integrity of the work as a whole. Article processing charges are supported by Storz Medical AG, Tägerwilen, Switzerland.

Conflict of interest. The principal investigator $\mathrm{K}$. Knobloch has been a speaker for Storz Medical AG (Tägerwilen, Switzerland) after the end of this randomized-controlled trial on various indications for ESWT which had no influence on this RCT.

B. Joest, R. Krämer, and P. M. Vogt declare no conflict of interest.

No company had any financial or intellectual influence on the design, the execution or the results of this RCT.

Compliance with ethics guidelines. This RCT was approved May 22, 2009 by the ethics institutional review board at Hannover Medical School, Germany, under the German title "Stosswellentherapie und Krafttraining zur Therapie der Cellulite-eine randomsiertkontrollierte Studie" (Nr. 5206). The study is internationally registered at ClinicalTrials.gov with ClinicalTrials.gov identifier: NCT00947414.
All procedures followed were in accordance with the ethical standards of the responsible committee on human experimentation (institutional and national) and with the Helsinki Declaration of 1975, as revised in 2000 and 2008. Informed consent was obtained from all patients for being included in the study and for the publication of patient photographs.

Open Access. This article is distributed under the terms of the Creative Commons Attribution Noncommercial License which permits any noncommercial use, distribution, and reproduction in any medium, provided the original author(s) and the source are credited.

\section{REFERENCES}

1. Proebstle TM. Cellulite. Hautarzt. 2010;61(10):864-72.

2. Emanuele E, Minoretti P, Altabas K, Gaeta E, Altabas V. Adiponectin expression in subcutaneous adipose tissue is reduced in women with cellulite. Int J Dermatol. 2011;50(4):412-6.

3. Hexsel D, Hexsel CL, Weber MB. Social impact of cellulite and its impact on quality of life. In: Goldman MP, Hexsel D, editors. Cellulite. Pathophysiology and treatment. 2nd ed. London: Informa Healthcare; 2010.

4. Christ C, Brenke G, Sattler G, et al. Steigerung der Hautelastizität und Revitalisierung der Dermis bei Cellulite und Bindegewebsschwäche durch die extrakorpoale Acoustic Wave Therapy (AWT). Ästhetische Dermatologie. 2008;1:2-10.

5. Sattler G, Pohl U, Raegener K. Pilotstudie akustische Wellentherapie (AWT) bei Cellulite. Äathetische Dermatologie. 2008;2:16-25.

6. Bae H, Kim J. Clinical outcomes of extracorporeal shock wave therapy in patients with secondary lymphedema: a pilot study. Ann Rehabil Med. 2013;37(2):229-34.

7. Tinazzi E, Amelio E, Marangoni E, et al. Effects of shock wave therapy in the skin of patients with 
progressive systemic sclerosis: a pilot study. Rheumatol Int. 2011;31:651-6.

8. Siems W, Grune T, Voss P, Brenke R. Anti-fibrosclerotic effects of shock wave therapy in lipedema and cellulite. BioFactors. 2005;24(1-4):275-82.

9. Angehrn F, Kuhn C, Voss A. Can cellulite be treated with low-energy extracorporal shock wave therapy? Clin Interv Aging. 2007;2(4):623-30.

10. Kuhn C, Angehrn F, Sonnabend O, Voss A. Impact of extracorporal shock waves on the human skin with cellulite: a case study of an unique instance. Clin Interv Aging. 2008;3(1):201-10.

11. Adatto M, Adatto-Neilson R, Servant JJ, Vester J, Novak P, Krotz A. Controlled, randomized study evaluating the effects of treating cellulite with $\mathrm{AWT}^{\circledR} / \mathrm{EPAT}^{\circledR}$. J Cosmet Laser Ther. 2010; 12:176-82.

12. CONSORT: http://www.consort-statement.org (Last accessed November 14, 2013).

13. Schulz KF, Altman DG, Moher D, for the CONSORT group. CONSORT statement: updated guidelines for reporting parallel group randomized trials. BMC Med. 2010;2010(8):18.

14. Knobloch K, Joest B, Vogt PM. Cellulite and extracorporeal shockwave therapy (CelluShock 2009) - a randomized trial. BMC Womens Health. 2010;10:29.

15. Hexsel DM, Dal'Forno TD, Hexsel CL. A validated photonumeric cellulite severity scale. J Eur Acad Dermatol Venereol. 2009;23(5):523-8.

16. Lautenschläger $H$. Skin diagnosis_based on measuring results. Kosmetik Int. 2008;10:54-6.

17. Lautenschläger H. Skin analysis-customer interview to complement measurements. Kosmetik Int. 2004;8:72-4.

18. Lautenschläger H. Skin analysis-with the support of modern instruments. Kosmetik Int. 2003;3:102-4.

19. Lautenschläger $H$. Instruments for skin analysis. Kostmetik Int. 2001;1:90.
20. Schulz KF, Chalmers I, Grimes DA, Altman DG. Assessing the quality of randomization from reports of controlled trials published in obstetrics and gynecology journals. JAMA. 1994;272:125-8.

21. Hexsel DM, Abreu M, Rodrigues TC, Soirefmann M, do Prado DZ, Gamboa MM. Side-by-side comparison of areas with and without cellulite depressions using magnetic resonance imaging. Dermatol Surg. 2009;35(10):1471-7.

22. Kim IG, Lee JY, Lee DS, Kwon JY, Hwang JH. Extracorporeal shockwave therapy combined with vascular endothelial growth factor-C hydrogel for lymphangiogenesis. J Vasc Res. 2013;50(2):124-33.

23. Raabe O, Shell K, Goessl A, et al. Effect of extracorporeal shock wave on proliferation and differentiation of equine adipose tissue-derived mesenchymal stem cells in vitro. Am J Stem Cells. 2013;2(1):62-73.

24. Tinazzi E, Amelio E, Marangoni E, et al. Effects of shock wave therapy in the skin of patients with progressive systemic sclerosis: a pilot study. Rheumatol Int. 2011;31(5):651-6.

25. Russe-Wilflingseder K, Russe E, Vester JC, Haller G, Novak P, Krotz A. Placebo controlled, prospectively randomized, double-blinded study for the investigation of the effectiveness and safety of the acoustic wave therapy (AWT) for cellulite treatment. J Cosmet Laser Ther. 2013;15(3):155-62.

26. Jackson RF, Roche GC, Shanks SC. A double-blind, placebo-controlled randomized trial evaluating the ability of low-level laser therapy to improve the appearance of cellulite. Lasers Surg Med. 2013;45(3):141-7.

27. Truitt A, Elkeeb L, Ortiz A, Saedi N, Echague A, Kelly KM. Evaluation of a long pulsed 1064-nm Nd:YAG laser for improvement in appearance of cellulite. J Cosmet Laser Ther. 2012;14(3):139-44.

28. Mlosek RK, Wozniak W, Malinowska S, Lewandowski M, Nowicki A. The effectiveness of anticellulite treatment using tripolar radiofrequency monitored by classic and highfrequency ultrasound. J Eur Acad Dermatol Venerol. 2012;26(6):696-703. 